\title{
RESEARCH
}

Open Access

\section{Subjective cognitive decline and subsequent dementia: a nationwide cohort study of 579,710 people aged 66 years in South Korea}

Yeong Chan Lee ${ }^{1,2+}$, Jae Myeong Kang ${ }^{3+}$, Hyewon Lee ${ }^{1,4+}$, Kiwon Kim ${ }^{5}$, Soyeon Kim ${ }^{1,2}$, Tae Yang Yu ${ }^{6}$, Eun-Mi Lee Clara Tammy Kim ${ }^{8}$, Doh Kwan Kim ${ }^{9}$, Matthew Lewis ${ }^{10}$, Hong-Hee Won ${ }^{2_{+}+}$, Frank Jessen ${ }^{11}$ and Woojae Myung ${ }^{{ }^{* *}}$

\begin{abstract}
Background: Subjective cognitive decline $(\mathrm{SCD})$ is a potential risk factor for dementia. We aimed to investigate the association between SCD and subsequent dementia in a nationwide population-based cohort in South Korea.

Methods: This cohort included 579,710 66-year-old adults who were followed for a total of 3,870,293 person-years (average $6.68 \pm 1.33$ years per person). All subjects completed a questionnaire about subjective memory impairment, the Pre-screening Korean Dementia Screening Questionnaire (KDSQ-P), which included a validated 5-item derivative, and were determined to have SCD based on a single question assessing memory decline. Depressive symptoms were assessed in all subjects using a 3-item modified geriatric depression scale. Hazard ratios were estimated using the Cox proportional hazards model and compared between subjects with and without SCD.

Results: Compared to subjects without SCD, those with SCD were more likely to develop dementia (incidence per 1000 person-years: non-SCD, 5.66; SCD, 8.59). After adjusting for potential confounding factors, the risk of subsequent dementia significantly increased in subjects with SCD, with an adjusted hazard ratio (aHR) of 1.38 (95\% confidence interval [CI] 1.34 to 1.41). The risk of subsequent dementia was greatly increased in subjects with higher KDSQ-P scores (aHR $=2.77,95 \% \mathrm{Cl} 2.35$ to 3.27). A significant association between SCD and dementia was observed in both depressive and non-depressive symptom groups ( $\mathrm{aHR}=1.50,95 \% \mathrm{Cl} 1.42$ to 1.57 in subjects with depressive symptoms; $\mathrm{aHR}=1.33,95 \% \mathrm{Cl} 1.29$ to 1.37 in subjects without depressive symptoms; $P=0.001$ ).

(Continued on next page)
\end{abstract}

\footnotetext{
* Correspondence: wonhh@skku.edu; wjmyung@snubh.org

†Yeong Chan Lee, Jae Myeong Kang, and Hyewon Lee are individuals who contributed equally to this work as co-first authors.

${ }^{2}$ Samsung Advanced Institute for Health Sciences and Technology (SAIHST), Sungkyunkwan University, Samsung Medical Center, 81 Irwon-ro,

Gangnam-gu, Seoul 06351, Republic of Korea

'Department of Neuropsychiatry, Seoul National University Bundang Hospital

Hospital, 29 Gumi-ro 173 Beon-gil, Bundang-gu, Seongnam-si 13619,

Gyeonggi-do, Republic of Korea

Full list of author information is available at the end of the article
}

(c) The Author(s). 2020 Open Access This article is licensed under a Creative Commons Attribution 4.0 International License, which permits use, sharing, adaptation, distribution and reproduction in any medium or format, as long as you give appropriate credit to the original author(s) and the source, provide a link to the Creative Commons licence, and indicate if changes were made. The images or other third party material in this article are included in the article's Creative Commons. licence, unless indicated otherwise in a credit line to the material. If material is not included in the article's Creative Commons licence and your intended use is not permitted by statutory regulation or exceeds the permitted use, you will need to obtain permission directly from the copyright holder. To view a copy of this licence, visit http://creativecommons.org/licenses/by/4.0/ The Creative Commons Public Domain Dedication waiver (http://creativecommons.org/publicdomain/zero/1.0/) applies to the data made available in this article, unless otherwise stated in a credit line to the data. 
(Continued from previous page)

Conclusions: In this population of 66-year-old individuals, SCD was significantly associated with an increased risk of subsequent dementia. This association was found in both depressive and non-depressive groups, with an increased risk of dementia in the presence of depressive symptoms. Our findings suggest that SCD indicates a risk for dementia. Further studies are needed to delineate potential approaches to preventing the development of dementia in individuals with SCD.

Keywords: Subjective cognitive decline, Subjective memory impairment, Alzheimer's disease, Dementia, Depression, Preclinical Alzheimer's disease

\section{Background}

Dementia represents one of the most prevalent neurodegenerative disorders worldwide and is present in approximately $10 \%$ of people aged 65 years and older [1]. Moreover, the public health burden of dementia is growing more rapidly than any other disease [2]. It has been reported that almost $80 \%$ of people worry about developing dementia [3] and identify dementia as their most feared illness, over cancer, heart disease, stroke, and diabetes [4]. Aging is concomitant with increased episodes of forgetfulness, and memory complaints are prevalent in approximately 25 to $50 \%$ of older adults [5]. Previous studies have found that memory complaints may relate to subclinical psychiatric symptoms [5-7], but they can also represent an early indicator of dementia, including Alzheimer's disease (AD) [8-12]. Considering the growing number of patients with dementia and the associated medical and societal burden, it is important to characterize at-risk groups or preclinical states of dementia in order to facilitate early interventions to reduce cognitive impairment in the future.

As part of this effort, considerable progress has been made investigating the prospective dementia risk associated with subjective cognitive decline (SCD) [13, 14]. $\mathrm{SCD}$ refers to a subjective experience of cognitive decline without objective cognitive deficits [14]. Research indicates that SCD may represent an early symptom of AD signifying the preclinical stage [9-12], which can progress to mild cognitive impairment (MCI) and dementia in the AD continuum [12-15]. However, SCD is also associated with poor physical health and with psychiatric disorders such as depression, which confounds the association with dementia [16, 17]. For example, depression is a major risk factor for dementia, and previous work has indicated that SCD could be linked with subsyndromal depressive symptoms rather than with subsequent dementia [18]. The considerable heterogeneity present across numerous small studies has contributed to conflicting results and has prevented consensus in the field [16-18].

Regarding the recruitment setting, it has been observed that SCD in memory clinic cases increased the risk of dementia [10], whereas SCD in community populations showed less significant or non-significant associations $[10,19]$. Given that the concerns and healthseeking behaviors of community-recruited older adults may differ from those of memory clinic patients [10], investigations using a large community sample would improve the accuracy for estimating the SCD-associated risk for incident dementia in the general population.

This study analyzed a nationwide population-based cohort that includes $51.8 \%$ of the 66-year-old adult population in South Korea. We aimed (1) to determine whether the risk of subsequent dementia increases in subjects with SCD compared to those without SCD, (2) to evaluate whether the severity of subjective memory impairment is associated with subsequent dementia, and (3) to examine whether depressive symptoms affect the association between SCD and subsequent dementia.

\section{Methods}

\section{Data sources and study cohort}

Data were obtained from the South Korean National Health Insurance Service (NHIS) database (supplementary methods) [20]. The NHIS provides mandatory healthcare for $97 \%$ of South Koreans under a singlepayer model. Further, it provides the National Screening Program for Transitional Ages (NSPTA), an age-specific national health examination program for all Korean citizens aged 40 and 66 [21]. Our study population consisted of a subset of individuals from the NHIS database who participated in the NSPTA at age 66, between 2009 and 2011. The study population covered $51.8 \%$ of the total South Korean population aged 66 during the enrolment period. This study was approved by the Institutional Review Board of Seoul National University Bundang Hospital. Because the NHIS provided encrypted data to protect private information, the need to obtain informed consent was waived (approval No. X1901-517-902).

\section{Inclusion and exclusion criteria}

We included all subjects who had available information on the Pre-screening Korean Dementia Screening Questionnaire (KDSQ-P), a cognitive function questionnaire [22]. The exclusion criteria were as follows: 
individuals who reported impaired function in activities of daily living (ADLs) because of possible pre-existing dementia (ADLs were assessed by a questionnaire consisting of six questions derived from a modified Korean Activities of Daily Living Scale and Korean Instrumental Activities of Daily Living Scale [23]); (2) individuals with dementia (ICD-10 [International Classification of Disease, 10th revision] code F00-F03, G30, or G31), mild cognitive impairment (ICD-10 code F06.7), or documented history of dementia medication (donepezil, rivastigmine, galantamine, or memantine) before the index date; (3) individuals with a psychotic disorder (ICD-10 code F20-F29; these criteria were applied to approximately meet the research criteria for pre-mild cognitive impairment SCD suggested in a previous study [14]); (4) individuals with missing or duplicate data on the primary variable of interest or covariates; (5) individuals with outlier values in continuous variables (mean \pm 4 standard deviations); and (6) individuals who died or dropped out between the time they participated in the NSPTA and the index date. From the 650,861 subjects who took the NSPTA, 71,150 subjects (10.9\%) were excluded according to the exclusion criteria (see Figure S1 in the online supplement).

\section{Primary independent variable of interest: SCD}

SCD was defined as an answer of "yes" to item 2 of the KDSQ-P. KDSQ-P is a validated questionnaire that consists of five questions [22]. Each item can be selfanswered with three possible choices: "no," "sometimes yes," or "frequently yes," scored as 0,1 , and 2, respectively. Overall KDSQ-P scores range from 0 to 10 , with higher scores indicating greater degrees of subjective memory impairment. Those who score $\geq 4$ points are advised to seek further evaluation of their cognitive function. All items of the KDSQ-P are presented in Table S1 in the online supplement. We defined SCD based on scoring a 1 or 2 (a positive answer) on the responses to item 2, which asks about the subjective decline in the memory domain and was suggested in the conceptual framework for research on SCD: "Do you think your memory has declined compared to a year ago?" [14].

\section{Subjective memory impairment and depressive symptoms}

The severity of the subjective memory impairment was defined using the total score on the KDSQ-P ranging from 0 to 10 [22]. The presence of depressive symptoms was defined as a Depression Screening Questionnaire (DSQ) score $>0$. This questionnaire includes three questions derived from a modified geriatric depression scale [24] (e.g., "Have you lost much of your activity or motivation these days?," "Do you feel that you are worthless now?," and "Do you feel that you have no hope now?").
Each question can be self-answered with two possible choices, "yes" or "no," scored as 1 or 0 , respectively. Total DSQ scores range from 0 to 3 , with higher scores indicating more depressive symptoms.

\section{Primary outcomes}

The primary outcome was the incidence of dementia following SCD. Dementia was defined based on the International Statistical Classification of Diseases, 10th revision codes (F00-F03, G30, or G31; Table S2) and the use of cognitive-enhancing medications based on previous studies $[25,26]$. This definition is relevant to our study considering that the Korean government covers medical expenditure for dementia based on ICD-10 codes. Additionally, clinicians are required to document ICD-10 codes for dementia as well as the results of neuropsychological tests to prescribe cognitiveenhancing medications. The date of onset of dementia was considered the first date for which patients were both diagnosed with dementia and prescribed with dementia medication.

\section{Covariates}

We assessed demographic variables such as sex and income. Lifestyle variables such as smoking status, alcohol consumption habits, and exercise frequency were included as covariates. We further adjusted for healthcare visit frequency, laboratory test results, physical examination results, and the patient's medical history, including information related to psychiatric disorders, neurological diseases, and other medical diseases (Table S2). To assess depressive symptoms, we used the DSQ score. Detailed information is presented in the supplementary methods.

\section{Statistical analysis}

To investigate the association between SCD and the incidence of dementia, study participants were followed from the index date (1 January of the year after each participant participated in the NSPTA) to the date of onset of dementia, death, or the end of follow-up (31 December 2017), whichever occurred first. For all participants, between-group differences for continuous variables and categorical variables were assessed using $t$ tests and chi-squared tests, respectively. A Cox proportional hazard regression analysis was conducted to determine adjusted hazard ratios (aHRs) for SCD in predicting subsequent dementia, after controlling for covariates. The effect of SCD on subsequent dementia was first analyzed in an unadjusted model and then in a sexadjusted and three additional models adjusted for various covariates (models 1 to 3 ). In the secondary analysis, we used the total KDSQ-P score as an independent variable to evaluate the association between the severity of 
subjective memory impairment and subsequent dementia. We also calculated aHR separately for score 1 and score 2 for each item of the KDSQ-P and the aHR of score 2 compared to score 1 only in the SCD group. We used an SCD and depressive symptom (coded as dichotomous variables: 0 indicating the absence of any depressive symptoms and 1 indicating the presence of any depressive symptoms among the three DSQ items) interaction term to test the potential for an interaction effect on subsequent dementia.

The proportional hazards assumption was graphically tested and verified using the Schoenfeld residual method. No variables violated the proportional hazards assumption. Multicollinearity between all covariates was tested using a variance inflation factor (VIF), and no significant collinearity was found (VIF $<4$ for all variables). After conducting a survival analysis of all participants, we performed an additional analysis by sampling the control group using the propensity score matching method based on logistic regression [27] using the Matchit packages in $\mathrm{R}$ (http://cran.r-project.org). We also performed several sensitivity analyses to confirm the robustness of the main findings. Firstly, we excluded patients who developed dementia within a year of the index date because those patients may not be incident cases. Secondly, we conducted separate analyses by dementia subtypes (AD and non- $\mathrm{AD}$ cases) to examine whether the association between SCD and dementia differs by dementia subtypes. Thirdly, we excluded patients with a history of psychiatric disorders, patients with a history of neurological diseases, or patients with depressive symptoms according to the DSQ (DSQ $>0$ ), because the presence of these disorders/diseases may confound the association of SCD with dementia affecting the dementia risk. Finally, we excluded patients with a KDSQ$P$ score $\geq 4$ (the cutoff point for further dementia screening tests [22]) to more rigorously exclude preexisting dementia cases.

Statistical analyses were conducted using two-tailed tests, a significance level of 0.05 , and $95 \%$ confidence intervals (CIs). All analyses were conducted using SAS Enterprise Guide version 7.2 (SAS Institute, Inc.) and R Studio version 1.0.136 (RStudio, Inc., with packages Survival version 2.43-3 and Survminer version 0.4.3).

\section{Results}

During the period from 2009 to 2011, a total of 650,861 individuals participated in the NSPTA and had KDSQ-P information available. Of these, we excluded 21,458 individuals who reported impaired ADL function; 12,658 individuals with dementia, MCI, or with a documented history of cognitive-enhancing medication; 18,760 individuals with missing or duplicate data; 14,315 individuals with outlier data; 2632 individuals with a psychotic disorder; and 1328 individuals who died or were lost to follow-up between their NSPTA participation date and the index date. In total, 579,710 subjects were included in the final study population for analysis, of which 222, 056 (38.3\%) experienced SCD (see the flowchart of study participants in Figure S1 in the online supplement). They were followed for an average of $6.68 \pm 1.33$ years per person and a total of 3,870,293 person-years.

\section{Subject characteristics}

The clinical and demographic characteristics of the participants at baseline are presented in Table 1 . The study population consisted of 266,311 (45.9\%) men and 313 , 399 (54.1\%) women. Compared to individuals in the non-SCD group, those with SCD tended to be women, did not smoke, consumed more alcohol, exercised more, visited healthcare facilities more frequently, had more medical or medication history, had higher cholesterol levels, and had lower fasting glucose, hemoglobin, and blood pressure.

\section{Risk of subsequent dementia according to SCD}

Among individuals with SCD, the incidence of dementia was 8.59 per 1000 person-years, which was higher than individuals without SCD who developed dementia at an incidence of 5.66 per 1000 person-years (Table 2). The SCD group had a higher cumulative incidence of dementia compared to the non-SCD group (log-rank $P<0.001$, Fig. 1). When adjusted for clinical factors (model 3), subjects with SCD had an increased risk of subsequent dementia $(\mathrm{aHR}=1.38,95 \%$ CI 1.34 to 1.41 in model 3; see Table 2). The aHRs were consistent in both men and women across all Cox regression models tested, despite controlling for various covariates. The effect of interaction between SCD and sex on subsequent dementia was not significant (Table S3 in the online supplement). The propensity score-matched analysis also confirmed that the presence of SCD increased the risk of subsequent dementia $(\mathrm{aHR}=1.39,95 \% \mathrm{CI} 1.36$ to 1.43 in model 3). The incidence of AD and dementia other than AD during the follow-up period is presented in Table S2 in the online supplement. The incidence rates of dementia associated with other risk factors including smoking, alcohol consumption, exercise frequency, depression, cerebrovascular disease, and diabetes mellitus are presented in Table S4 in the online supplement.

\section{Association between severity of subjective memory impairment and subsequent dementia}

The severity of subjective memory impairment, total KDSQ-P score, was significantly associated with risk of subsequent dementia (Fig. 2). Subjects with a higher KDSQ-P score showed a strong tendency for a higher risk for subsequent dementia. The risk of dementia in 
Table 1 Descriptive characteristics of the study population

\begin{tabular}{|c|c|c|c|c|}
\hline & Total $(n=579,710)$ & Non-SCD group $(n=357,654)$ & SCD group $(n=222,056)$ & $P$ value \\
\hline Sex & & & & $<0.0001$ \\
\hline Male & $266,311(45.9 \%)$ & $173,795(48.6 \%)$ & $92,516(41.7 \%)$ & \\
\hline Female & $313,399(54.1 \%)$ & $183,859(51.4 \%)$ & $129,540(58.3 \%)$ & \\
\hline Income & & & & $<0.0001$ \\
\hline Medicaid aid & $33,023(5.7 \%)$ & $19,101(5.3 \%)$ & $13,922(6.3 \%)$ & \\
\hline Group 1 (1st to 6th ventiles) & $125,064(21.6 \%)$ & $82,491(23.1 \%)$ & $42,573(19.2 \%)$ & \\
\hline Group 2 (7th to 14 th ventiles) & $178,625(30.8 \%)$ & $110,550(30.9 \%)$ & $68,075(30.7 \%)$ & \\
\hline Group 3 (15th to 20th ventiles) & $242,998(41.9 \%)$ & $145,512(40.7 \%)$ & $97,486(43.9 \%)$ & \\
\hline \multicolumn{5}{|l|}{ Lifestyle factors } \\
\hline Smoking status & & & & $<0.0001$ \\
\hline Never smoked & $406,103(70.1 \%)$ & $250,238(70.0 \%)$ & $155,865(70.2 \%)$ & \\
\hline Ex-smoker & $95,502(16.5 \%)$ & $57,647(16.1 \%)$ & $37,855(17.0 \%)$ & \\
\hline Current smoker & $78,105(13.5 \%)$ & 49,769 (13.9\%) & $28,336(12.8 \%)$ & \\
\hline Alcohol consumption & & & & $<0.0001$ \\
\hline No drinking: rarely & $508,547(87.7 \%)$ & $314,324(87.9 \%)$ & $194,223(87.5 \%)$ & \\
\hline Light drinking: 3-4 times per week & $38,840(6.7 \%)$ & $23,954(6.7 \%)$ & $14,886(6.7 \%)$ & \\
\hline Heavy drinking: almost every day & $32,323(5.6 \%)$ & $19,376(5.4 \%)$ & $12,947(5.8 \%)$ & \\
\hline Exercise frequency & & & & $<0.0001$ \\
\hline Exercise & $327,775(56.5 \%)$ & $200,723(56.1 \%)$ & $127,052(57.2 \%)$ & \\
\hline No exercise & $251,935(43.5 \%)$ & $156,931(43.9 \%)$ & $95,004(42.8 \%)$ & \\
\hline \multicolumn{5}{|l|}{ Healthcare visit frequency* } \\
\hline First quartile & $144,858(25.0 \%)$ & $93,542(26.2 \%)$ & $51,316(23.1 \%)$ & \\
\hline Second quartile & $144,945(25.0 \%)$ & $89,583(25.1 \%)$ & $55,362(24.9 \%)$ & \\
\hline Third quartile & $144,988(25.0 \%)$ & $88,208(24.7 \%)$ & $56,780(25.6 \%)$ & \\
\hline Fourth quartile & $144,919(25.0 \%)$ & $86,321(24.1 \%)$ & $58,598(26.4 \%)$ & \\
\hline \multicolumn{5}{|l|}{ Past medical history } \\
\hline \multicolumn{5}{|l|}{ Psychiatric disorders } \\
\hline Depression & $48,653(8.4 \%)$ & $26,676(7.5 \%)$ & 21,977 (9.9\%) & $<0.0001$ \\
\hline Bipolar affective disorder & $1891(0.3 \%)$ & 1059 (0.3\%) & $832(0.4 \%)$ & $<0.0001$ \\
\hline Substance use disorder & $3522(0.6 \%)$ & $2035(0.6 \%)$ & $1487(0.7 \%)$ & $<0.0001$ \\
\hline Panic disorder & $2315(0.4 \%)$ & $1298(0.4 \%)$ & $1017(0.5 \%)$ & $<0.0001$ \\
\hline Obsessive-compulsive disorder & $671(0.1 \%)$ & $370(0.1 \%)$ & $301(0.1 \%)$ & 0.001 \\
\hline Personality disorder & $237(0.0 \%)$ & $142(0.0 \%)$ & $95(0.0 \%)$ & 0.619 \\
\hline Other psychiatric disorders & $140,212(24.2 \%)$ & $81,764(22.9 \%)$ & $58,448(26.3 \%)$ & $<0.0001$ \\
\hline \multicolumn{5}{|l|}{ Neurological diseases } \\
\hline Cerebrovascular disease & $80,212(13.8 \%)$ & $46,895(13.1 \%)$ & $33,317(15.0 \%)$ & $<0.0001$ \\
\hline Epilepsy & $8622(1.5 \%)$ & 4807 (1.3\%) & 3815 (1.7\%) & $<0.0001$ \\
\hline Migraines & $43,466(7.5 \%)$ & $25,304(7.1 \%)$ & $18,162(8.2 \%)$ & $<0.0001$ \\
\hline Headaches & 70,207 (12.1\%) & 40,791 (11.4\%) & 29,416 (13.2\%) & $<0.0001$ \\
\hline Sleep disorder & 63,769 (11.0\%) & 36,635 (10.2\%) & $27,134(12.2 \%)$ & $<0.0001$ \\
\hline Head injury & $64,698(11.2 \%)$ & 39,459 (11.0\%) & 25,239 (11.4\%) & $<0.0001$ \\
\hline \multicolumn{5}{|l|}{ Medical diseases } \\
\hline Diabetes mellitus & $154,977(26.7 \%)$ & $94,346(26.4 \%)$ & $60,631(27.3 \%)$ & $<0.0001$ \\
\hline Myocardial infarction & $8504(1.5 \%)$ & $5245(1.5 \%)$ & $3259(1.5 \%)$ & 0.981 \\
\hline
\end{tabular}


Table 1 Descriptive characteristics of the study population (Continued)

\begin{tabular}{|c|c|c|c|c|}
\hline & Total $(n=579,710)$ & Non-SCD group $(n=357,654)$ & $\operatorname{SCD}$ group $(n=222,056)$ & $P$ value \\
\hline Congestive heart failure & $31,952(5.5 \%)$ & $19,222(5.4 \%)$ & $12,730(5.7 \%)$ & $<0.0001$ \\
\hline Liver disease & $146,020(25.2 \%)$ & $88,392(24.7 \%)$ & $57,628(26.0 \%)$ & $<0.0001$ \\
\hline Renal disease & $5669(1.0 \%)$ & $3476(1.0 \%)$ & $2193(1.0 \%)$ & 0.564 \\
\hline Peptic ulcer disease & $259,797(44.8 \%)$ & $155,929(43.6 \%)$ & $103,868(46.8 \%)$ & $<0.0001$ \\
\hline Thyroid gland disorder & $40,236(6.9 \%)$ & $23,104(6.5 \%)$ & $17,132(7.7 \%)$ & $<0.0001$ \\
\hline Asthma & $123,850(21.4 \%)$ & $74,598(20.9 \%)$ & $49,252(22.2 \%)$ & $<0.0001$ \\
\hline Cancer & $41,290(7.1 \%)$ & $24,845(6.9 \%)$ & $16,445(7.4 \%)$ & $<0.0001$ \\
\hline \multicolumn{5}{|l|}{ Medication history } \\
\hline HMG-CoA reductase inhibitors & $128,527(22.2 \%)$ & $77,725(21.7 \%)$ & $50,802(22.9 \%)$ & $<0.0001$ \\
\hline Diabetes medication & $84,015(14.5 \%)$ & $51,878(14.5 \%)$ & $32,137(14.5 \%)$ & 0.735 \\
\hline Antihypertensive medication & $288,262(49.7 \%)$ & $178,093(49.8 \%)$ & $110,169(49.6 \%)$ & 0.180 \\
\hline Antidepressants & $31,079(5.4 \%)$ & $17,216(4.8 \%)$ & $13,863(6.2 \%)$ & $<0.0001$ \\
\hline Benzodiazepines and sleeping pills & $92,444(15.9 \%)$ & $53,108(14.8 \%)$ & $39,336(17.7 \%)$ & $<0.0001$ \\
\hline Antiplatelet medication & $140,615(24.3 \%)$ & $86,071(24.1 \%)$ & $54,544(24.6 \%)$ & $<0.0001$ \\
\hline Depression Screening Questionnaire score, mean (SD) & $0.34(0.79)$ & $0.23(0.67)$ & $0.53(0.92)$ & $<0.0001$ \\
\hline \multicolumn{5}{|l|}{ Laboratory findings } \\
\hline \multicolumn{5}{|l|}{ Cholesterol level, mean (SD), mg/dL } \\
\hline LDL cholesterol & $117.47(35.58)$ & $117.26(35.55)$ & $117.81(35.64)$ & $<0.0001$ \\
\hline HDL cholesterol & $53.63(13.64)$ & $53.57(13.61)$ & $53.73(13.68)$ & $<0.0001$ \\
\hline Triglycerides & $134.31(70.64)$ & $134.80(70.97)$ & $133.54(70.10)$ & $<0.0001$ \\
\hline Fasting glucose & $101.89(20.96)$ & $102.11(21.10)$ & $101.52(20.73)$ & $<0.0001$ \\
\hline Hemoglobin & $13.59(1.40)$ & $13.63(1.40)$ & $13.54(1.39)$ & $<0.0001$ \\
\hline \multicolumn{5}{|l|}{ Physical examination findings } \\
\hline Body mass index & $24.29(2.98)$ & $24.31(2.99)$ & $24.25(2.98)$ & $<0.0001$ \\
\hline Systolic blood pressure & $128.74(15.43)$ & $129.05(15.44)$ & $128.22(15.41)$ & $<0.0001$ \\
\hline Diastolic blood pressure & $78.01(9.73)$ & $78.18(9.73)$ & $77.73(9.73)$ & $<0.0001$ \\
\hline
\end{tabular}

$H D L$ high-density lipoprotein, $L D L$ low-density lipoprotein, $S C D$ subjective cognitive decline, $S D$ standard deviation

*The fourth quartile group had the highest frequency of medical visits

subjects with a score of 9 or 10 was approximately three times higher than in subjects with a score of 0. Each item in the KDSQ-P was also significantly associated with the risk of subsequent dementia (see Table S1 in the online supplement). Subjects who answered "frequently yes" for each question had a higher risk for subsequent dementia than those who answered "sometimes yes."

\section{Effect of interaction between SCD and depressive symptoms on subsequent dementia}

Figure 3 shows the estimated effect of SCD on subsequent dementia after accounting for depressive symptoms. Regardless of the presence of depressive symptoms, SCD was significantly associated with a risk for subsequent dementia. Notably, the effect of SCD on subsequent dementia was particularly prominent in the presence of depressive symptoms (in subjects with depressive symptoms, $\mathrm{aHR}=1.50,95 \%$ CI 1.42 to 1.57 ; in subjects without depressive symptoms, aHR $=1.33,95 \%$ CI 1.29 to 1.37; interaction $P=0.001$ ).

\section{Sensitivity analysis for the association between SCD and subsequent dementia}

Even after iteratively removing subgroups from our subjects, the risk of subsequent dementia was consistently associated with SCD (see Table S5 in the online supplement). First, we excluded patients diagnosed with subsequent dementia within 1 year of the index date to avoid the onset of actual dementia before reporting SCD. By excluding these patients, our analysis demonstrated the robustness of the link between SCD and subsequent dementia in our study cohort $(\mathrm{aHR}=1.37,95 \%$ CI 1.34 to 1.41 in model 3). The results were also consistent when subsequent dementia was subdivided into $\mathrm{AD}$ (only including $\mathrm{AD}, \mathrm{aHR}=1.38,95 \% \mathrm{CI} 1.35$ to 1.42 in model 3 ) and dementia other than $\mathrm{AD}$ (only including dementia other than $\mathrm{AD}, \mathrm{aHR}=1.37,95 \% \mathrm{CI} 1.30$ to 1.45 in model 
Table 2 Cox regression analysis for the association between subjective cognitive decline and subsequent dementia

\begin{tabular}{|c|c|c|}
\hline & Non-SCD group & SCD group \\
\hline Total population & $357,654(61.7 \%)$ & $222,056(38.3 \%)$ \\
\hline Dementia events & $13,501(3.8 \%)$ & $12,766(5.8 \%)$ \\
\hline Person-years & $2,384,745$ & $1,485,548$ \\
\hline Incidence (events/1000 person-years) & 5.66 & 8.59 \\
\hline Unadjusted HR (95\% Cl) & 1 [reference] & $1.51(1.47-1.55)$ \\
\hline Sex-adjusted HR (95\% Cl) & 1 [reference] & $1.48(1.44-1.51)$ \\
\hline aHR in model $1(95 \% \mathrm{Cl})^{*}$ & 1 [reference] & $1.46(1.43-1.50)$ \\
\hline aHR in model $2(95 \% \mathrm{Cl})^{\dagger}$ & 1 [reference] & $1.42(1.39-1.46)$ \\
\hline aHR in model $3(95 \% \mathrm{Cl})^{\ddagger}$ & 1 [reference] & $1.38(1.34-1.41)$ \\
\hline Men & $173,795(48.6 \%)$ & $92,516(41.7 \%)$ \\
\hline Dementia events & $5480(3.2 \%)$ & $4399(4.8 \%)$ \\
\hline Person-years & $1,147,608$ & $611,069.20$ \\
\hline Incidence (events/1000 person-years) & 4.78 & 7.20 \\
\hline Unadjusted HR (95\% Cl) & 1 [reference] & $1.50(1.44-1.56)$ \\
\hline aHR in model $1(95 \% \mathrm{Cl})^{*}$ & 1 [reference] & $1.49(1.43-1.55)$ \\
\hline aHR in model $2(95 \% \mathrm{Cl})^{\dagger}$ & 1 [reference] & $1.44(1.39-1.50)$ \\
\hline aHR in model $3(95 \% \mathrm{Cl})^{\ddagger}$ & 1 [reference] & $1.38(1.32-1.44)$ \\
\hline Women & $183,859(51.4 \%)$ & $129,540(58.3 \%)$ \\
\hline Dementia events & $8021(4.4 \%)$ & $8367(6.5 \%)$ \\
\hline Person-years & $1,237,137$ & $874,478.80$ \\
\hline Incidence (events/1000 person-years) & 6.48 & 9.57 \\
\hline Unadjusted HR (95\% Cl) & 1 [reference] & $1.47(1.42-1.51)$ \\
\hline aHR in model $1(95 \% \mathrm{Cl})^{*}$ & 1 [reference] & $1.45(1.40-1.49)$ \\
\hline aHR in model $2(95 \% \mathrm{Cl})^{+}$ & 1 [reference] & $1.41(1.37-1.45)$ \\
\hline aHR in model $3(95 \% \mathrm{Cl})^{\ddagger}$ & 1 [reference] & $1.38(1.33-1.42)$ \\
\hline
\end{tabular}

aHR adjusted hazard ratio, $\mathrm{Cl}$ confidence interval, $S C D$ subjective cognitive decline

*Adjusted for sex, income, lifestyle factors, and healthcare visit frequency (in subgroup analysis for men and women, sex was not entered as a covariate)

${ }^{\dagger}$ Adjusted for sex, income, lifestyle factors, healthcare visit frequency, medical history, and medication history (in subgroup analysis for men and women, sex was not entered as a covariate)

${ }^{\ddagger}$ Adjusted for sex, income, lifestyle factors, healthcare visit frequency, medical history, medication history, depression screening questionnaire scores, laboratory

findings, and physical examination findings (in subgroup analysis for men and women, sex was not entered as a covariate)

3). The definition and the incidence of dementia other than AD are presented in Table S2. We also observed a significant link after excluding individuals with psychiatric disorders $(\mathrm{aHR}=1.38,95 \% \mathrm{CI} 1.34$ to 1.43 in model 3 ), patients with neurological diseases ( $\mathrm{aHR}=1.43,95 \%$ CI 1.38 to 1.49 in model 3), patients with depressive symptoms according to the DSQ ( $\mathrm{aHR}=1.33,95 \% \mathrm{CI}$ 1.29 to 1.37 in model 3), and patients with KDSQ-P scores $\geq 4$ (aHR $=1.18,95 \%$ CI 1.14 to 1.21 in model 3). In addition, the association between the severity of SCD (score 1 or 2 to item 2) and the risk of dementia remained significant after excluding the non-SCD group (score 0 to item 2) (Table S6 in the online supplement).

\section{Discussion}

In this nationwide population-based study of 579,710 66-year-old adults, subjects with SCD were more likely to develop subsequent dementia than those without SCD over an average follow-up period of 6.68 years. The association between SCD and subsequent dementia was robust across sex, subtype of dementia (AD or other than $\mathrm{AD}$ ), history of psychiatric disorders or neurological diseases, and presence of depressive symptoms. The severity of subjective memory impairment was also associated with the risk of subsequent dementia. Furthermore, regardless of the presence of depressive symptoms, SCD was significantly associated with subsequent dementia, with an increased association in the presence of depressive symptoms.

The positive association between SCD and subsequent dementia found in our study is generally consistent with previous studies. A recent population-based study $(n=$ 2710) reported an aHR in SCD similar to that of our study $(\mathrm{aHR}=1.18,95 \%$ CI 1.03 to 1.33) [19]. The 


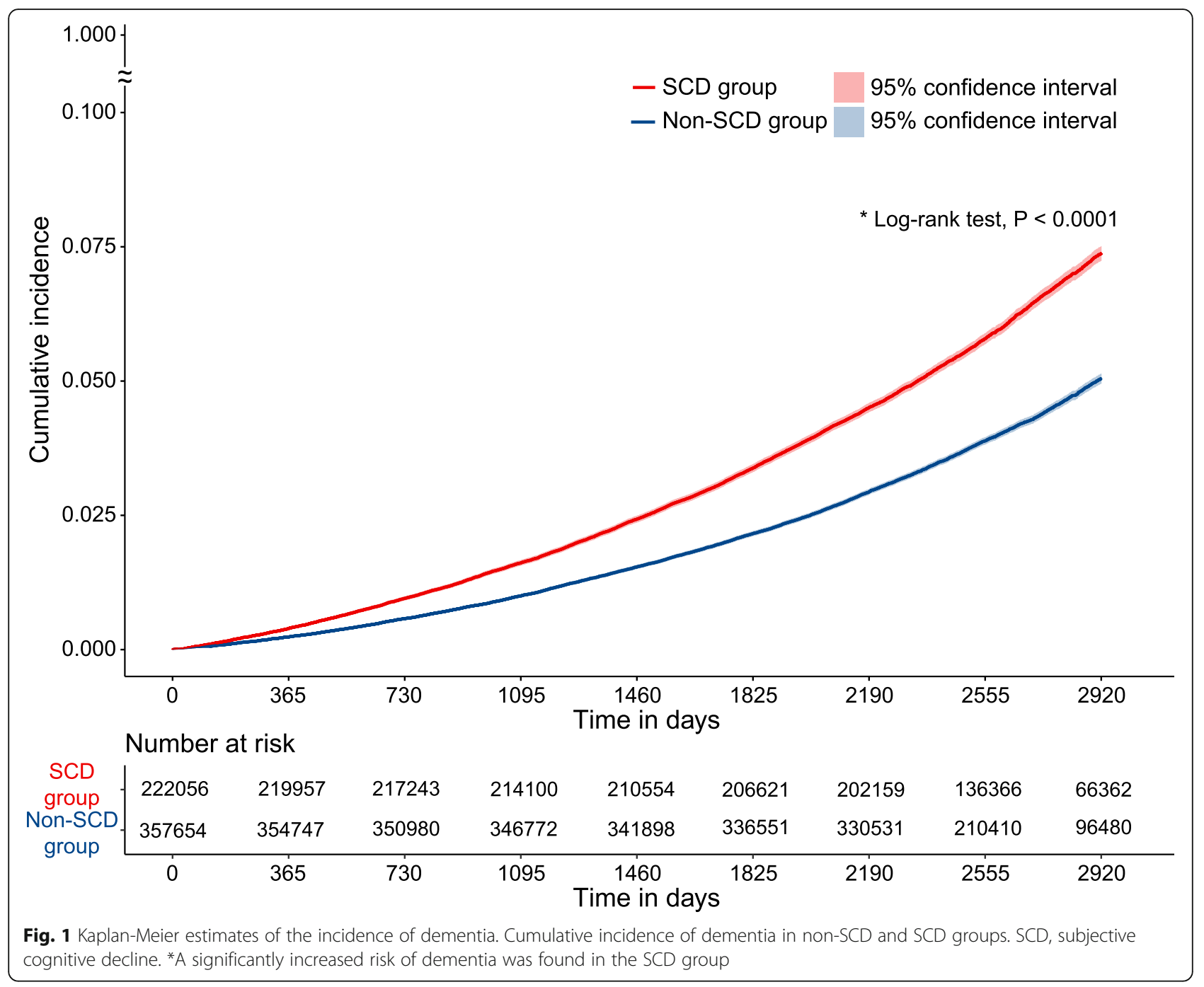

prevalence of SCD in our study was 38.3\% (222,056 in $579,710)$, which is comparable to the prevalence estimates of previous community-based studies, which ranged from 22.1 to $56.0 \%$ [5]. However, the rate of incident dementia and risk of subsequent dementia in the SCD group compared to the non-SCD group in our study was lower than in previous research. In a recent multicenter cohort study of 4369 participants, the incidence rate of dementia in SCD cases was reported to be 17.7 per 1000 person-years [10], which is higher than our result of 8.6 per 1000 person-years. Discrepancies between our results and those of previous SCD studies may be due to the heterogeneity of the study populations $[10,12]$. Reports have indicated that, when compared to community populations, patients who visited memory clinics had a higher progression rate from normal cognition to MCI [28], from SCD to AD [10], and from MCI to AD [29].

The higher progression rate observed in memory clinic samples has been attributed to the subjects' greater likelihood of experiencing the early signs of neurodegenerative diseases and of spontaneously reporting memory complaints $[9,10,14,28,29]$. More importantly, decreased functional abilities were found in memory clinic attendees at baseline, which significantly indicates a risk for dementia $[28,29]$. In contrast to previous studies, our study consisted of subjects obtained from a population-based setting, and thus better illustrates the robust association between SCD and subsequent dementia in the general population. In accordance with a previous study [19], our analyses revealed equivalent incidence rates of dementia associated with SCD (8.6 per 1000 person-years) and other risk factors including current smoking (7.24/1000 person-years), heavy alcohol drinking (7.44/1000 person-years), no exercise (7.54/1000 person-years), diabetes mellitus (8.91/1000 person-years), cerebrovascular disease $(11.34 / 1000$ person-years), and depression (12.46/1000 person-years). This result suggests that SCD has a similar magnitude of risk for subsequent dementia as other lifestyle and vascular risk factors in a community population. 


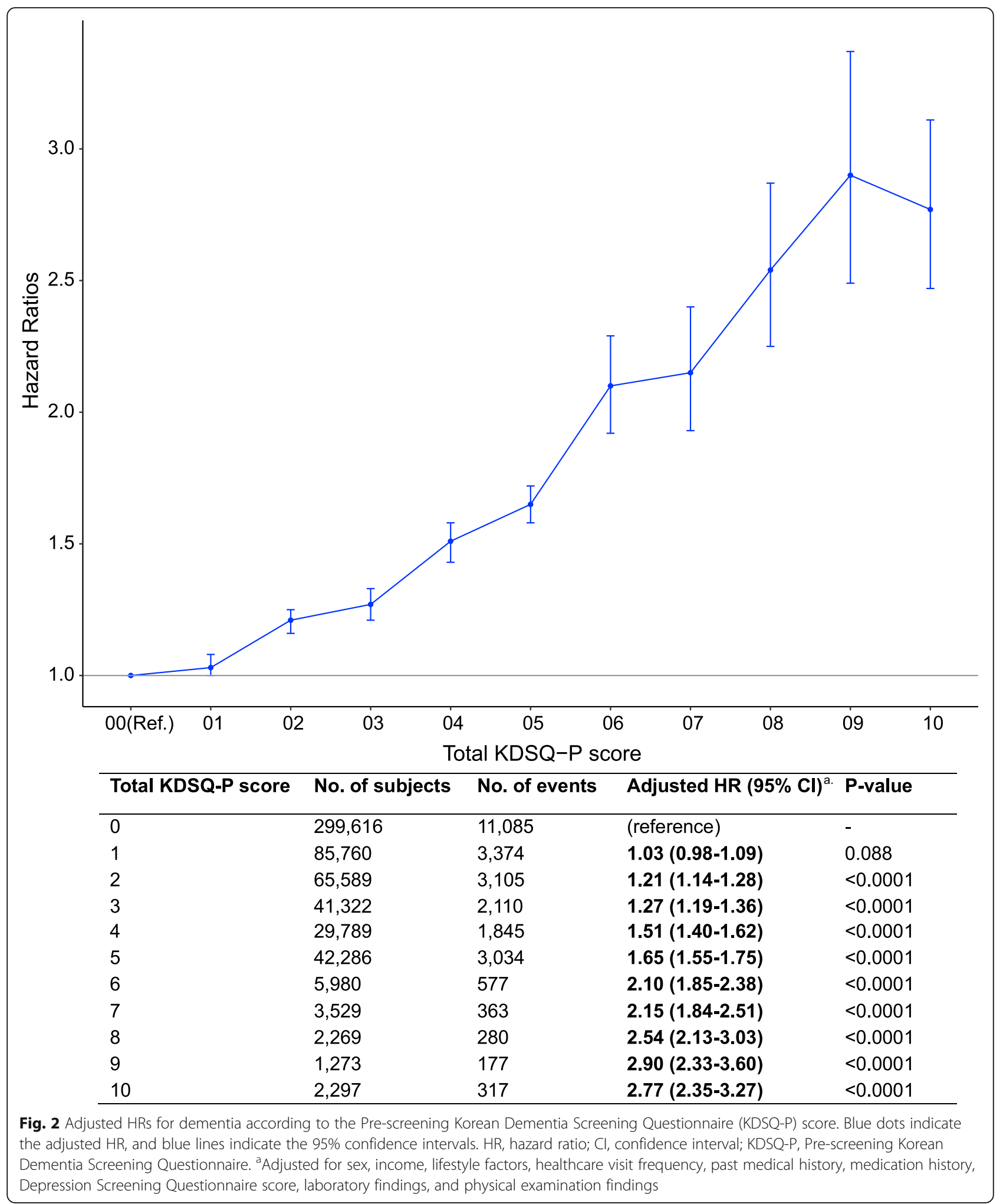

Our analysis showed a higher incidence of dementia in women with SCD than in men with SCD (9.57 vs. 7.20/ 1000 person-years), but the risk of dementia associated with SCD was comparable in both sexes after adjusting for various factors $(\mathrm{aHR}=1.38,95 \%$ CI 1.33 to 1.42 for women; $\mathrm{aHR}=1.38,95 \% \mathrm{CI} 1.32$ to 1.44 for men; Table 2 and Table S3 in the online supplement). Some studies reported women to be more susceptible than men to 


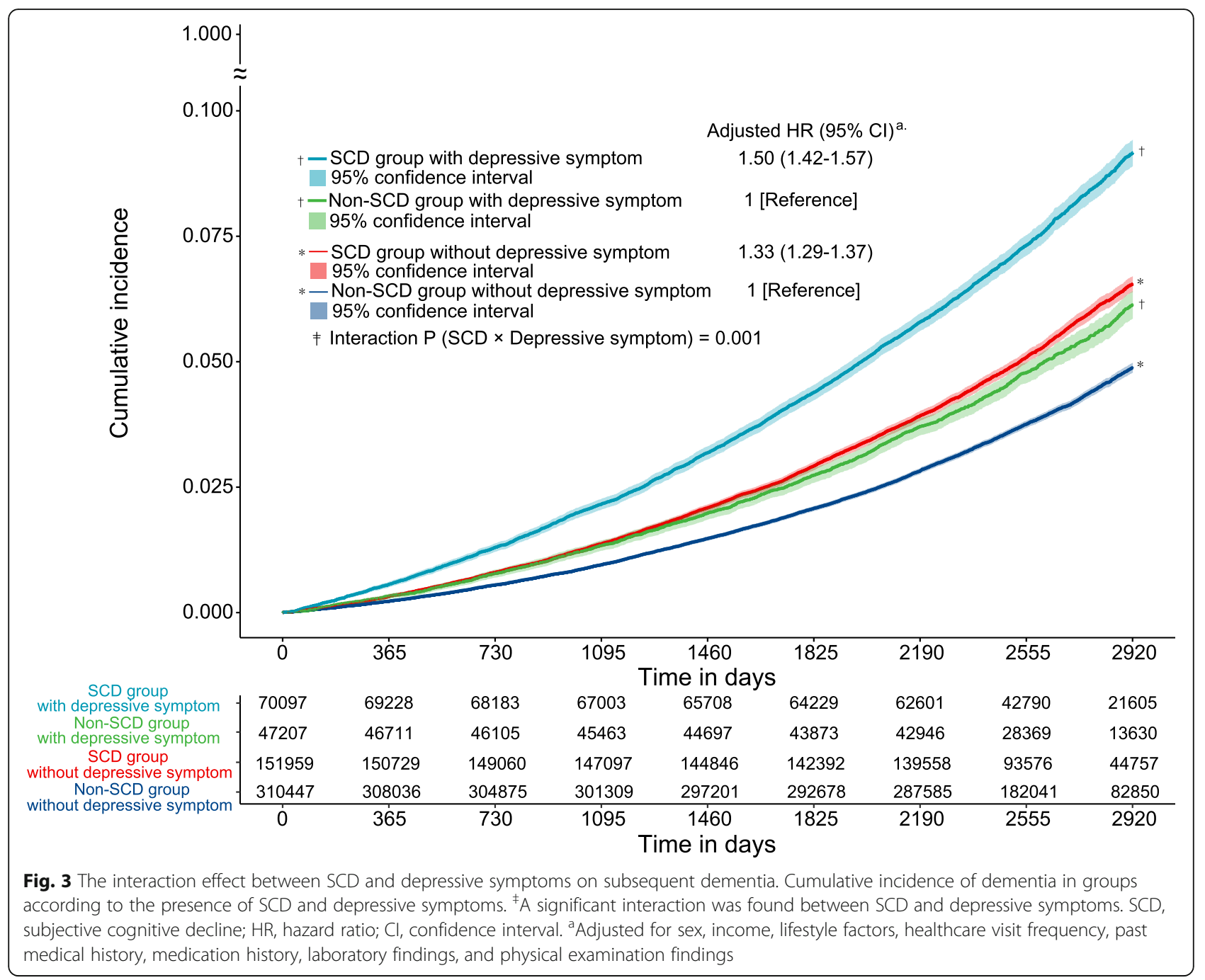

progression from SCD to dementia [30, 31], whereas others found no significant sex difference $[8,10,19]$. Some have reported a tendency for women to report SCD worries with a higher sensitivity to subtle cognitive symptoms relating to dementia progression when compared to men [32]. Women are also known to be susceptible to dementia, possibly due to their longevity and sex-specific biological factors [33]. However, in our study with the largest sample size, before and after adjusting for various clinical factors and sociodemographic variables, the risk of dementia associated with SCD was comparable in both sexes.

Our results also highlight the positive linear association between the severity of subjective memory impairment and subsequent dementia (Fig. 2). This finding suggests that the more severe the subjective memory complaints, the greater the risk of subsequent dementia. KDSQ-P, a validated pre-screening tool for dementia [22], includes items measuring subjective memory using multiple response types. In recent studies, many authors have evaluated SCD with multiple items [34, 35], and some have administered face-to-face interviews $[8,30,34,36]$. Moreover, many of them asked about specific memory (70.7\%) and functional decline (41.6\%) to assess SCD [34]. The single question defining SCD in this study lacked information on concerns, non-memory domains, and impairment. However, the use of a general question to identify the presence of SCD and a variety of additional questions regarding specific subjective memory impairment may also clarify the effect of well-defined features of SCD on subsequent dementia.

In this study, the SCD group with depressive symptoms had a greater risk for subsequent dementia than the group without depressive symptoms, with a significant interaction effect (Fig. 3). Although depressive symptoms are regarded as a crucial factor for subsequent dementia due to their association with cognitive disorders $[7,13,16,37]$, previous studies have found a minimal effect of mood scores on the association between SCD and further cognitive decline $[8,19,38]$. This is 
possibly attributable to the limited size of the studies. Our results imply that SCD and depressive symptoms not only act independently as risk factors for dementia but also contribute to its development through their interaction.

We observed that SCD was likely to be an incipient symptom of both $\mathrm{AD}$ and non-AD-related dementias (see Table S5 in the online supplement). Studies have suggested that $\mathrm{SCD}$ is related to AD pathology. It has been demonstrated that $\mathrm{AD}$ biomarkers such as cerebrospinal fluid $\beta$ amyloid [39, 40], plasma $\beta$-amyloid [41], hippocampal atrophy [41], and amyloid retention in positron emission tomography [42] are associated with SCD. Although the prevalence of $\mathrm{AD}$ pathology in $\mathrm{SCD}$ may differ between memory centers due to their varied study designs [43], SCD might be an early symptom in the preclinical stage of $\mathrm{AD}$. Previous studies have reported inconsistent results regarding the association between SCD and non-AD dementia, such as vascular dementia, Lewy body dementia, and frontotemporal lobar degeneration $[8,10]$. Although the typical symptoms of dementia differ according to the case, memory dysfunction could represent an early symptom in all forms of dementia [44]. Importantly, memory dysfunction can have diverse manifestations including difficulties with episodic and semantic memory and encoding, retrieval, and recognition types of memory. Our results suggest that $S C D$ can broadly be used as a risk indicator for a myriad of cognitive disorders such as $\mathrm{AD}$ and non-AD.

The major strength of our study is that we have used the largest nationwide representative cohort data to date relating SCD to subsequent dementia. We analyzed 579 , 710 eligible subjects, extracted from over 50 million entries in the NHIS database. Clinical cohorts in SCD research have relatively small to modest numbers of selective participants, ranging from 42 to 4500 [10, 34]. In addition, studies that have assessed the risks associated with subjective memory complaints have used diverse and inconsistent characteristics, including the number of participants (17 to 2901), the age of participants (18 to 87 ), the follow-up periods (1 to 15 years), the operational criteria for defining $\mathrm{SCD}$, and the methods of assessing dementia [6, 12, 34]. Consequently, when these studies are combined for meta-analysis, the significant heterogeneity between studies may add significant noise towards estimating the association between SCD and dementia. As an additional strength, our results are based on the mandatory national healthcare screening service, which is more reflective of the general population and might be more robust and generalizable than studies conducted through memory clinics. In this study, measuring SCD in a large homogeneous community population with comprehensive information enabled us to investigate $\mathrm{SCD}$ and risk for both $\mathrm{AD}$ and non- $\mathrm{AD}$ dementia with a wide range of clinical covariates, extended time frame, consideration of depressive disorder and subclinical symptoms, and comparison with peers of the same age without SCD.

This study also has several limitations. Firstly, the main weakness is the lack of objective cognition test results. Normal performance on standardized cognitive tests is one of the research criteria for SCD [14]. To reduce bias related to this limitation, we excluded subjects with pre-existing cognitive decline from the analysis, namely subjects with impaired ADLs, a documented history of dementia, MCI, or a prescription for dementia medication. Secondly, although we comprehensively adjusted for various confounds, we did not consider the years of education, occupational attainment, family history, imaging biomarkers, or other potentially relevant confounds. However, we adjusted for covariates such as comprehensive disease diagnosis, income level, and healthcare visit frequency that can only be obtained from the national data. Thirdly, the operational definition of AD may be susceptible to misdiagnosis or underdiagnosis, although the incidence rate of $\mathrm{AD}$ in our study population was similar to the rates reported in epidemiological studies conducted in South Korea [45]. Fourthly, the age of 66 years of this cohort is relatively young, and thus, the findings may not represent the entire elderly group. Finally, because the study population included individuals from only a single country, our findings may not be generalizable to people of other backgrounds.

\section{Conclusion}

Our study, in a population-based cohort, is the largest to date and demonstrates the importance of SCD as an early, independent risk factor for dementia. These findings thus provide strong evidence for the role of SCD in characterizing the initial high-risk stage of dementia. As a growing public health issue, SCD should be further investigated as a risk factor for dementia. Giving additional attention to SCD as a risk factor for dementia could facilitate more focused surveillance from the public and healthcare professionals. However, it may not be appropriate for the public to view SCD as a disease state that should be actively treated. Instead, an approach focused on prevention for people with $\mathrm{SCD}$, including lifestyle modifications or providing education on dementia, could be promising. Future studies should further explore the clinical and neurobiological nature of SCD as an early sign of dementia.

\section{Supplementary information}

Supplementary information accompanies this paper at https://doi.org/10. 1186/s13195-020-00618-1.

Additional file 1. Supplementary methods, tables, figures, and references. 


\section{Abbreviations}

AD: Alzheimer's disease; ADLs: Activities of daily living; aHR: Adjusted hazard ratio; Cl: Confidence interval; DSQ: Depression Screening Questionnaire; KDSQ-P: Korean Dementia Screening Questionnaire; MCl: Mild cognitive impairment; NHIS: National Health Insurance Service; NSPTA: National Screening Program for Transitional Ages; SCD: Subjective cognitive decline

\section{Acknowledgements}

Not applicable

\section{Authors' contributions}

YCL and HL conceived and designed the study, acquired and analyzed the data, interpreted the study findings, and drafted the manuscript. JMK conceived and designed the study; defined the exclusion criteria and exposure, outcome, and covariate categories; interpreted the study findings; and drafted the manuscript. KK, SK, TYY, EML, CTK, and DKK designed the study; defined the exclusion criteria and exposure, outcome, and covariate categories; interpreted the study findings; and drafted the manuscript. HHW, FJ, and WM conceived and designed the study, interpreted the study findings, supervised and directed the conduct of the study, and critically reviewed the manuscript. The corresponding authors attest that all listed authors meet the authorship criteria and that no others meeting the criteria have been omitted. HHW and WM are the guarantors of the work. YCL, JMK, and $\mathrm{HL}$ contributed equally to this work as co-first authors. HHW (wonhh@skku.edu) and WM (wjmyung@snubh.org) contributed equally to this work and should be considered as co-corresponding authors. The authors read and approved the final manuscript.

\section{Funding}

This work was supported by a National Research Foundation (NRF) of Korea Grant, funded by the Korean government (NRF-2018R1C1B6001708; WM); the Basic Science Research Program through the NRF of Korea, funded by the Ministry of Education (NRF-2018R1D1A1B07049034; HL); and an NRF grant funded by the Korean government (MSIT, NRF-2019R1A2C4070496; HHW). The funding sources had no role in the study design; collection, analysis, and interpretation of the data; writing of the report; or decision to submit the article for publication. All researchers are independent of relationships with the funders, and all authors, external and internal, had full access to the study's data and can take responsibility for the integrity of the data and the accuracy of the data analysis.

\section{Availability of data and materials}

This study is based on the National Health Insurance Service (NHIS) register data in South Korea (NHIS-2019-1-211). Because these data belong to the $\mathrm{NHIS}$, the authors are not permitted to share them, except in aggregate (as, for example, in a publication). However, interested parties can obtain the data on which the study was based by submitting a research protocol to the NHIS (https://nhiss.nhis.or.kr/bd/ab/bdaba000eng.do). The analytic/statistical codes are available from the corresponding author (wjmyung@snubh.org, WM), upon reasonable request.

\section{Ethics approval and consent to participate}

This study was approved by the Institutional Review Board of Seoul National University Bundang Hospital. Because the National Health Insurance Service (NHIS) provided encrypted data to protect private information, the need to obtain informed consent was waived (approval No. X-1901-517-902).

\section{Consent for publication}

Not applicable

\section{Competing interests}

All authors have completed the International Committee of Medical Journal Editors (ICMJE) uniform disclosure form at www.icmje.org/coi_disclosure.pdf (available on request from the corresponding author) and declare no support from any organization for the submitted work, no financial relationships with any organizations that might have a competing interest in the submitted work in the previous three years, and no other relationships or activities that could appear to have influenced the submitted work.

\section{Author details}

Department of Neuropsychiatry, Seoul National University Bundang Hospital Hospital, 29 Gumi-ro 173 Beon-gil, Bundang-gu, Seongnam-si 13619, Gyeonggi-do, Republic of Korea. ${ }^{2}$ Samsung Advanced Institute for Health Sciences and Technology (SAIHST), Sungkyunkwan University, Samsung Medical Center, 81 Irwon-ro, Gangnam-gu, Seoul 06351, Republic of Korea. ${ }^{3}$ Department of Psychiatry, Gil Medical Center, Gachon University College of Medicine, Incheon, Republic of Korea. ${ }^{4}$ Institute of Health and Environment, Seoul National University, Seoul, Republic of Korea. ${ }^{5}$ Department of Psychiatry, Veteran Health Service Medical Center, Seoul, Republic of Korea. ${ }^{6}$ Division of Endocrinology and Metabolism, Department of Medicine, Wonkwang Medical Center, Wonkwang University School of Medicine, Iksan, Republic of Korea. 'Department of Health Science, Dongduk Women's University, Seoul, Republic of Korea. ${ }^{8}$ Institute of Life and Death Studies, Hallym University, Chuncheon, Gangwon-do, Republic of Korea. ${ }^{9}$ Department of Psychiatry, Samsung Medical Center, Sungkyunkwan University School of Medicine, Seoul, Republic of Korea. ${ }^{10}$ The Department of General Practice, Melbourne Medical School, The University of Melbourne, Melbourne, Victoria, Australia. ${ }^{11}$ Department of Psychiatry, University of Cologne, Cologne, Germany.

Received: 13 December 2019 Accepted: 13 April 2020 Published online: 06 May 2020

\section{References}

1. Hauer ME. Population projections for U.S. counties by age, sex, and race controlled to shared socioeconomic pathway. Scientific Data. 2019;6:190005

2. Nichols E, Szoeke CE, Vollset SE, Abbasi N, Abd-Allah F, Abdela J, et al. Global, regional, and national burden of Alzheimer's disease and other dementias, 1990-2016: a systematic analysis for the Global Burden of Disease Study 2016. Lancet Neurology. 2019;18:88-106.

3. International AsD. World Alzheimer report 2019: attitudes to dementia. Alzheimer's Disease Internationals London; 2019.

4. Anderson LA, Day KL, Beard RL, Reed PS, Wu B. The public's perceptions about cognitive health and Alzheimer's disease among the U.S. population: a national review. The Gerontologist. 2009;49:S3-S11.

5. Jonker C, Geerlings Ml, Schmand B. Are memory complaints predictive for dementia? A review of clinical and population-based studies. Int J Geriatric Psychiatry. 2000;15:983-91.

6. Reid LM, Maclullich AM. Subjective memory complaints and cognitive impairment in older people. Dement Geriatr Cogn Disord. 2006;22:471-85.

7. Grut M, Jorm AF, Fratiglioni L, Forsell Y, Viitanen M, Winblad B. Memory complaints of elderly people in a population survey: variation according to dementia stage and depression. J Am Geriatr Soc. 1993;41:1295-300.

8. Jessen F, Wiese B, Bachmann C, Eifflaender-Gorfer S, Haller F, Kölsch H, et al. Prediction of dementia by subjective memory impairment: effects of severity and temporal association with cognitive impairment. Arch Gen Psychiatry. 2010;67:414-22.

9. Jessen F, Wolfsgruber S, Wiese B, Bickel H, Mösch E, Kaduszkiewicz H, et al. $\mathrm{AD}$ dementia risk in late $\mathrm{MCl}$, in early $\mathrm{MCl}$, and in subjective memory impairment. Alzheimer's Dement. 2014;10:76-83.

10. Slot RE, Sikkes SA, Berkhof J, Brodaty H, Buckley R, Cavedo E, et al. Subjective cognitive decline and rates of incident Alzheimer's disease and non-Alzheimer's disease dementia. Alzheimer's Dement. 2019;15:465-76.

11. Schofield PW, Marder K, Dooneief G, Jacobs DM, Sano M, Stern Y. Association of subjective memory complaints with subsequent cognitive decline in community-dwelling elderly individuals with baseline cognitive impairment. Am J Psychiatry. 1997;154:609-15.

12. Mitchell A, Beaumont H, Ferguson D, Yadegarfar M, Stubbs B. Risk of dementia and mild cognitive impairment in older people with subjective memory complaints: meta-analysis. Acta Psychiatr Scand. 2014;130:439-51.

13. Molinuevo JL, Rabin LA, Amariglio R, Buckley R, Dubois B, Ellis KA, et al. Implementation of subjective cognitive decline criteria in research studies. Alzheimer's Dement. 2017;13:296-311.

14. Jessen F, Amariglio RE, Van Boxtel M, Breteler M, Ceccaldi M, Chételat G, et al. A conceptual framework for research on subjective cognitive decline in preclinical Alzheimer's disease. Alzheimer's Dement. 2014;10:844-52.

15. Jack CR Jr, Bennett DA, Blennow K, Carrillo MC, Dunn B, Haeberlein SB, et al. NIA-AA Research Framework: toward a biological definition of Alzheimer's disease. Alzheimer's Dement. 2018;14:535-62. 
16. Markova H, Andel R, Stepankova H, Kopecek M, Nikolai T, Hort J, et al. Subjective cognitive complaints in cognitively healthy older adults and their relationship to cognitive performance and depressive symptoms. J Alzheimer's Disease. 2017:59:871-81.

17. Jorm AF, Butterworth P, Anstey KJ, Christensen H, Easteal S, Maller J, et al. Memory complaints in a community sample aged 60-64 years: associations with cognitive functioning, psychiatric symptoms, medical conditions, APOE genotype, hippocampus and amygdala volumes, and white-matter hyperintensities. Psychol Med. 2004;34:1495-506.

18. Mirza SS, Wolters FJ, Swanson SA, Koudstaal PJ, Hofman A, Tiemeier H, et al. 10-year trajectories of depressive symptoms and risk of dementia: a population-based study. Lancet Psychiatry. 2016;3:628-35.

19. Licher S, Leening MJ, Yilmaz P, Wolters FJ, Heeringa J, Bindels PJ, et al. Development and validation of a dementia risk prediction model in the general population: an analysis of three longitudinal studies. Am J Psychiatr. 2018;176:543-51.

20. Lee J, Lee JS, Park SH, Shin SA, Kim K. Cohort profile: the National Health Insurance Service-National Sample Cohort (NHIS-NSC). South Korea Int J Epidemiol. 2017:46:e15

21. Kim HS, Shin DW, Lee WC, Kim YT, Cho B. National screening program for transitional ages in Korea: a new screening for strengthening primary prevention and follow-up care. J Korean Med Sci. 2012;27:S70-S5.

22. Jeon Y, Yun K, Kim Y. Validation of KDSQ-P as selecting elderly for KDSQ-C Korean J Health Promot. 2010;10:45-52.

23. Won J, Yang K, Noh Y. The development of Korean Activities of Daily Living (K-ADL) and Korean Instrumental Activities of Daily Living (K-IADL) scale. J Korean Geriatrics Society. 2002;6:107-20.

24. Bae JN, Cho MJ. Development of the Korean version of the Geriatric Depression Scale and its short form among elderly psychiatric patients. J Psychosom Res. 2004;57:297-305.

25. Katon W, Pedersen HS, Ribe AR, Fenger-Grøn M, Davydow D, Waldorff FB, et al. Effect of depression and diabetes mellitus on the risk for dementia: a national population-based cohort study. JAMA Psychiatry. 2015;72:612-9.

26. Kim CT, Myung $W$, Lewis $M$, Lee $H$, Kim SE, Lee $K$, et al. Exposure to general anesthesia and risk of dementia: a nationwide population-based cohort study. J Alzheimer's Dis. 2018;63:395-405.

27. Rosenbaum PR, Rubin DB. Constructing a control group using multivariate matched sampling methods that incorporate the propensity score. Am Stat. 1985;39:33-8.

28. Chen Y, Denny KG, Harvey D, Farias ST, Mungas D, DeCarli C, et al. Progression from normal cognition to mild cognitive impairment in a diverse clinic-based and community-based elderly cohort. Alzheimer's Dementia. 2017;13:399-405.

29. Farias ST, Mungas D, Reed BR, Harvey D, DeCarli C. Progression of mild cognitive impairment to dementia in clinic- vs community-based cohorts. Arch Neurol. 2009;66:1151-7.

30. Heser K, Kleineidam L, Wiese B, Oey A, Roehr S, Pabst A, et al. Subjective cognitive decline may be a stronger predictor of incident dementia in women than in men. J Alzheimer's Dis. 2019;68:1469-78.

31. Pérès $K$, Helmer $C$, Amieva $H$, Matharan F, Carcaillon L, Jacqmin-Gadda $H$, et al. Gender differences in the prodromal signs of dementia: memory complaint and IADL-restriction. A prospective population-based cohort. J Alzheimer's Dis. 2011;27:39-47.

32. Sundermann EE, Edmonds EC, Delano-Wood L, Galasko DR, Salmon DP, Rubin $\mathrm{LH}$, et al. Sex influences the accuracy of subjective memory complaint reporting in older adults. J Alzheimer's Disease. 2018;61:1163-78.

33. Snyder HM, Asthana S, Bain L, Brinton R, Craft S, Dubal DB, et al. Sex biology contributions to vulnerability to Alzheimer's disease: a think tank convened by the Women's Alzheimer's Research Initiative. Alzheimer's Dementia. 2016; 12:1186-96.

34. Rabin LA, Smart CM, Crane PK, Amariglio RE, Berman LM, Boada M, et al. Subjective cognitive decline in older adults: an overview of self-report measures used across 19 international research studies. J Alzheimer's Dis. 2015;48:S63-86

35. van Harten AC, Mielke MM, Swenson-Dravis DM, Hagen CE, Edwards KK, Roberts $\mathrm{RO}$, et al. Subjective cognitive decline and risk of $\mathrm{MCl}$ : the Mayo Clinic Study of Aging. Neurology. 2018;91:e300-e12.

36. Miebach L, Wolfsgruber S, Polcher A, Peters O, Menne F, Luther K, et al. Which features of subjective cognitive decline are related to amyloid pathology? Findings from the DELCODE study. Alzheimer's Res Therapy. 2019;11:66.
37. Diniz BS, Butters MA, Albert SM, Dew MA, Reynolds CF. Late-life depression and risk of vascular dementia and Alzheimer's disease: systematic review and meta-analysis of community-based cohort studies. Br J Psychiatry. 2013; 202:329-35.

38. Reisberg B, Shulman MB, Torossian C, Leng L, Zhu W. Outcome over seven years of healthy adults with and without subjective cognitive impairment. Alzheimer's Dement. 2010;6:11-24.

39. Wolfsgruber S, Jessen F, Koppara A, Kleineidam L, Schmidtke K, Frölich L, et al. Subjective cognitive decline is related to CSF biomarkers of AD in patients with MCI. Neurology. 2015;84:1261-8.

40. Visser PJ, Verhey F, Knol DL, Scheltens P, Wahlund L-O, Freund-Levi Y, et al. Prevalence and prognostic value of CSF markers of Alzheimer's disease pathology in patients with subjective cognitive impairment or mild cognitive impairment in the DESCRIPA study: a prospective cohort study. Lancet Neurol. 2009;8:619-27.

41. Cantero JL, Iglesias JE, Van Leemput K, Atienza M. Regional hippocampal atrophy and higher levels of plasma amyloid-beta are associated with subjective memory complaints in nondemented elderly subjects. J Gerontol Series A. 2016;71:1210-5.

42. Amariglio RE, Becker JA, Carmasin J, Wadsworth LP, Lorius N, Sullivan C, et al. Subjective cognitive complaints and amyloid burden in cognitively normal older individuals. Neuropsychologia. 2012;50:2880-6.

43. Wolfsgruber S, Molinuevo JL, Wagner M, Teunissen CE, Rami L, Coll-Padrós $\mathrm{N}$, et al. Prevalence of abnormal Alzheimer's disease biomarkers in patients with subjective cognitive decline: cross-sectional comparison of three European memory clinic samples. Alzheimer's Res Ther. 2019;11:1-11.

44. Karantzoulis S, Galvin JE. Distinguishing Alzheimer's disease from other major forms of dementia. Expert Rev Neurother. 2011;11:1579-91.

45. Kim YJ, Han JW, So YS, Seo JY, Kim KY, Kim KW. Prevalence and trends of dementia in Korea: a systematic review and meta-analysis. J Korean Med Sci. 2014;29:903-12.

\section{Publisher's Note}

Springer Nature remains neutral with regard to jurisdictional claims in published maps and institutional affiliations.
Ready to submit your research? Choose BMC and benefit from:

- fast, convenient online submission

- thorough peer review by experienced researchers in your field

- rapid publication on acceptance

- support for research data, including large and complex data types

- gold Open Access which fosters wider collaboration and increased citations

- maximum visibility for your research: over $100 \mathrm{M}$ website views per year

At BMC, research is always in progress.

Learn more biomedcentral.com/submissions 